\title{
Investigation of the effects of nuclear level density parameters on the cross sections for the ${ }^{234} \mathrm{U}(\gamma, \mathrm{f})$ reaction
}

\author{
Hakan Pekdogan ${ }^{1, a}$, Abdullah Aydin ${ }^{1}$ and Ismail Hakki Sarpun ${ }^{2}$ \\ ${ }^{1}$ Kirikkale University, Faculty of Arts and Science, Department of Physics, Kirikkale, Turkey \\ ${ }^{2}$ Afyon Kocatepe University, Faculty of Arts and Science, Department of Physics, Afyonkarahisar, Turkey
}

\begin{abstract}
In this study, we have investigated the effects of nuclear level density parameters on the cross sections for the ${ }^{234} \mathrm{U}(\gamma, \mathrm{f})$ reaction up to $20 \mathrm{MeV}$. The cross sections on ${ }^{234} \mathrm{U}(\gamma, \mathrm{f})$ reaction were calculated for different level density models using the TALYS 1.6 code. First, it was determined the level density model that was the closest to the experimental data. Secondly, cross sections obtained for different level density parameters of this model were compared with experimental data from the EXFOR database. Thus it was determined the best level density parameter fit to experimental data.
\end{abstract}

\section{Introduction}

The level density and level density parameters are very important quantities for describing the structure properties of nuclei. Especially, the level density parameter is an essential ingredient as input for calculation of reaction cross sections. The calculated cross sections are important for practical applications such as the modeling of nuclear astrophysical processes (e.g. nucleosynthesis in different stellar environments), medical research, and reactor technology. Also knowledge of nuclear fission cross sections is very important for understanding of nuclear structure. There are many reliable computer program to calculate the fission cross sections. One of them is TALYS 1.6 nuclear code [1].

In the literature, there have been many studies on nuclear data evaluation for fission cross section and fission yield using different model approximation theoretically. For better nuclear data calculations, computer codes are used. TALYS, a software, has been developed to capture all nuclear reaction model calculation, allowing to provide nuclear reaction simulation from $1 \mathrm{keV}-1000 \mathrm{MeV}$ energy range more precisely. Nuclear reaction types that TALYS provided are nuclear reaction that involved neutron, proton, deuteron, photon, triton, ${ }^{3} \mathrm{He}$ and alpha particles as projectile and element of mass 12 and heavier as target [2].

Calculation result obtained from TALYS are depends on great input parameter tuning based on experimental value using curve fitting. At this point, experimental data play important role in calculation performed by TALYS to achieve better agreement with it $[3,4]$.

\footnotetext{
${ }^{a}$ Corresponding author: hakanpdogan@gmail.com
}

Besides that, to achieve even more accurate result, the proper selection of parameter adjustment and reaction mechanism should be implemented. This could be done by using theoretical prediction about the behavior of specific nuclear reaction. With proper selection of reaction parameter and mechanism combined with parameter tuning would lead to better approximated calculation result.

In this study the cross sections on ${ }^{234} \mathrm{U}(\gamma, \mathrm{f})$ reaction were calculated for different level density models using the TALYS 1.6 code [1]. All the calculations were compared with each other and with the experimental data obtained from EXFOR library [5].

\section{Level Densities}

Effective level densities had no explicit dependencies with nuclear collective effect.

\subsection{Constant Temperature Model (CTM)}

This model divides energy range into two parts, low energy part from $0 \mathrm{MeV}$ to the matching energy $\mathrm{E}_{\mathrm{m}}$ where Constant Temperature Law applied, and high energy part above $\mathrm{E}_{\mathrm{m}}$ where Fermi Gas Model applied [6].

\subsection{Back-Shifted Fermi Gas Model (BSFGM)}

In this model, Fermi gas expression is used in all energy range. As a consequence, pairing energy parameter should be adjustable [7]. 


\subsection{Generalized Superfluid Model (GSM)}

This Model takes superconductive pairing correlation into account based on Bardeen-Cooper-Schrieffer theory [8, 9].

\section{The level density parameter $a$}

A nuclide-specific constant value for the level density parameter a may be proposition for the form of word described and in fact the first level density analyses spanning an entire range of nuclides $[6,7,10]$ treated $a$ as a parameter independent of energy. After that, Ignatyuk et al. [11] the correlation in the middle the parameter $a$ and the shell correction term of the liquid-drop component of the mass formula was found. These researchers considered that a more reasonable level density is obtained by assuming Fermi gas formula given above is still valid. However, with energy-dependent expression for $a$, the inclusion of energy-dependent shell effects is important for the efficiency. The existence of shell effects at low energy and their disappearance at high energy in a phenomenological manner considered for its appearance [12].

It reads

$$
a=a\left(\mathrm{E}_{\mathrm{X}}\right)=\tilde{\mathrm{a}}\left(1+\delta \mathrm{W} \frac{1-\exp [-\gamma \mathrm{U}]}{\mathrm{U}}\right) .
$$

\section{Methods}

In this study, comparison have been realized between three level density models and experimental values taken from the EXFOR in $(\gamma, f)$ reaction for ${ }^{234} \mathrm{U}(\gamma, \mathrm{f})[5]$. The TALYS 1.6 code used in theoretical calculations created by Koning and his colleagues at NRG Petten, Netherlands and CEA Bruyéres-le-Châtel, France to provide a complete and accurate simulation of nuclear reactions involving neutrons, $\gamma$ rays, protons, deuterons, tritons, ${ }^{3} \mathrm{He}$, and alphas in the $1 \mathrm{keV}-1 \mathrm{GeV}$ energy range, through an optimal combination of reliable nuclear models. Nuclear structure and model parameters are implemented through Reference Input Parameter Library (RIPL, 1998) [13].

\section{Results}

In this work $(\gamma, f)$ reaction cross sections of ${ }^{234} \mathrm{U}(\gamma, \mathrm{f})$ were calculated for three level density models using TALYS 1.6 code in incident energy range of $4-20 \mathrm{MeV}$. The calculated results and available experimental data are presented in Figs. 1-4.

Table. 1. Level density parameter values used in CTM model calculations

\begin{tabular}{|c|c|c|c|c|c|}
\hline${ }^{234} \mathrm{U}(\gamma, \mathrm{f})$ & default & $\mathrm{a}-10 \mathrm{a} \%$ & $\mathrm{a}+10 \mathrm{a} \%$ & $\mathrm{a}-20 \mathrm{a} \%$ & $\mathrm{a}+20 \mathrm{a} \%$ \\
\hline $\mathrm{CTM}$ & 15.8128 & 17.3941 & 14.2315 & 18.97540 & 12.6502 \\
\hline
\end{tabular}

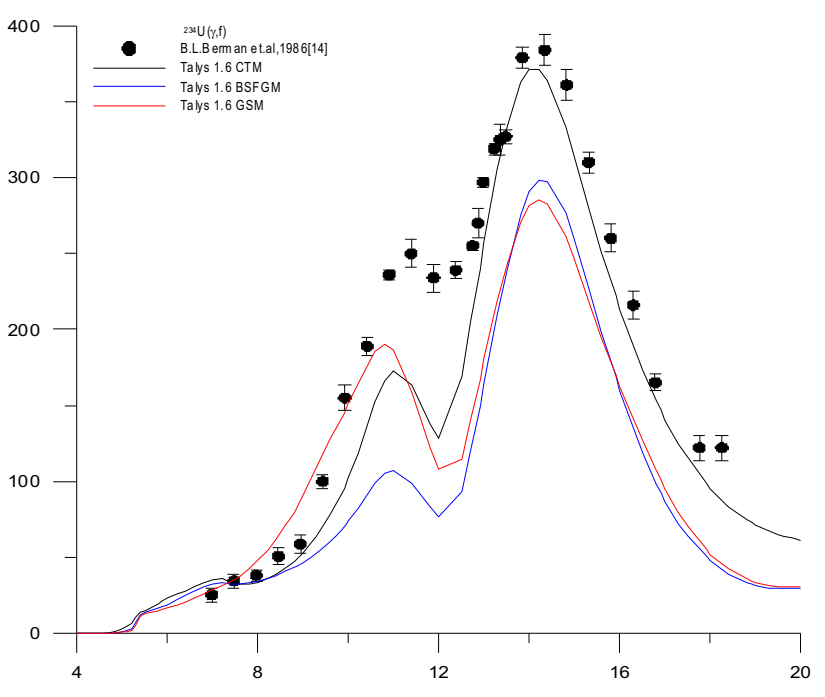

Figure 1. Theoretical calculations of ${ }^{234} \mathrm{U}(\gamma, \mathrm{f})$ reaction cross section using level density models in TALYS. The experimental data are taken from EXFOR.

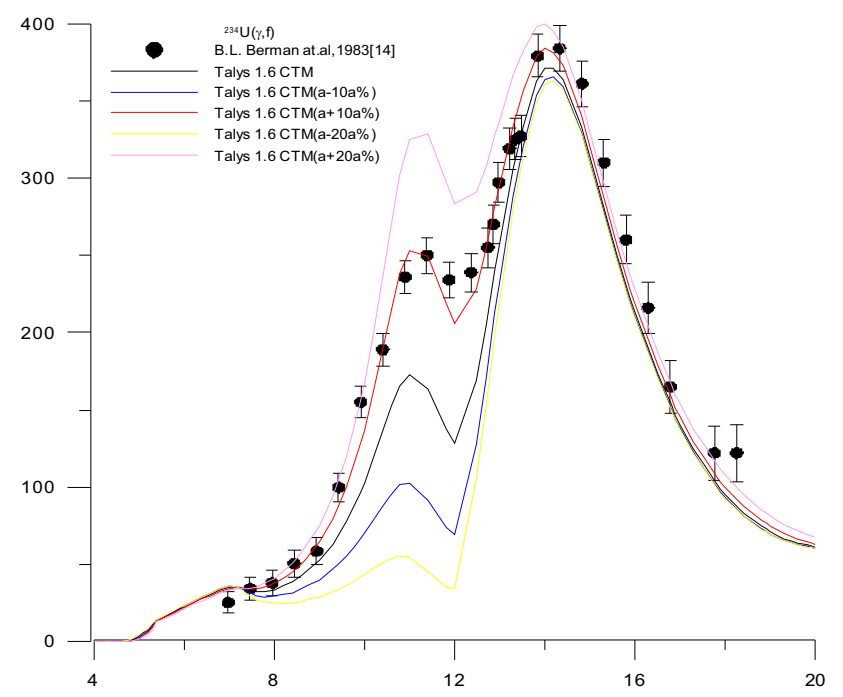

Figure. 2. Theoretical calculations of ${ }^{234} \mathrm{U}(\gamma, \mathrm{f})$ reaction cross section using CTM level density model. The experimental data are taken from EXFOR.

Table. 2. Level density parameter values used in BSFGM model calculations

\begin{tabular}{|l|l|l|l|l|l|l|}
\hline${ }^{234} \mathrm{U}(\gamma, \mathrm{f})$ & default & $\mathrm{a}-10 \mathrm{a} \%$ & $\mathrm{a}+10 \mathrm{a} \%$ & $\mathrm{a}-20 \mathrm{a} \%$ & $\mathrm{a}+20 \mathrm{a} \%$ & $\mathrm{a}+25 \mathrm{a} \%$ \\
\hline BSGFM & 12.9515 & 14.2466 & 11.6564 & 15.5418 & 10.3612 & 17.4845 \\
\hline
\end{tabular}

Table. 3. Level density parameter values used in GSM model calculations

\begin{tabular}{|l|l|l|l|l|l|l|}
\hline${ }^{234} \mathrm{U}(\gamma, \mathrm{f})$ & default & $\mathrm{a}+10 \mathrm{a} \%$ & $\mathrm{a}-10 \mathrm{a} \%$ & $\mathrm{a}+20 \mathrm{a} \%$ & $\mathrm{a}-20 \mathrm{a} \%$ & $\mathrm{a}+35 \mathrm{a} \%$ \\
\hline $\mathrm{GSM}$ & 13.4189 & 14.7608 & 12.0770 & 16.1027 & 10.7351 & 18.1155 \\
\hline
\end{tabular}




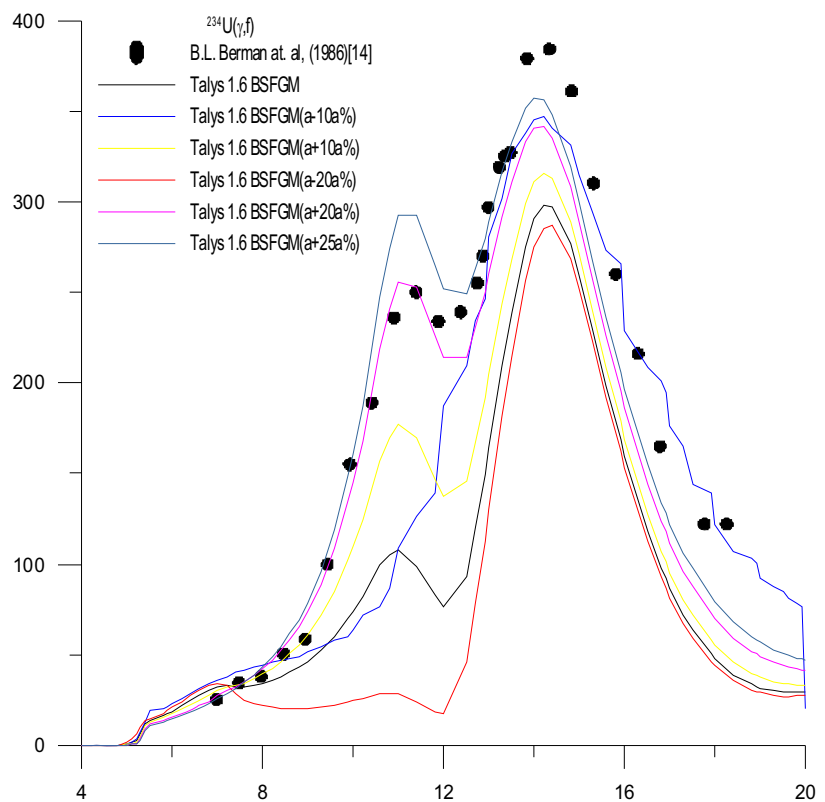

Fig. 3. Theoretical calculations of ${ }^{234} \mathrm{U}(\gamma, \mathrm{f})$ reaction cross section using BSFGM level density model. The experimental data are taken from EXFOR.

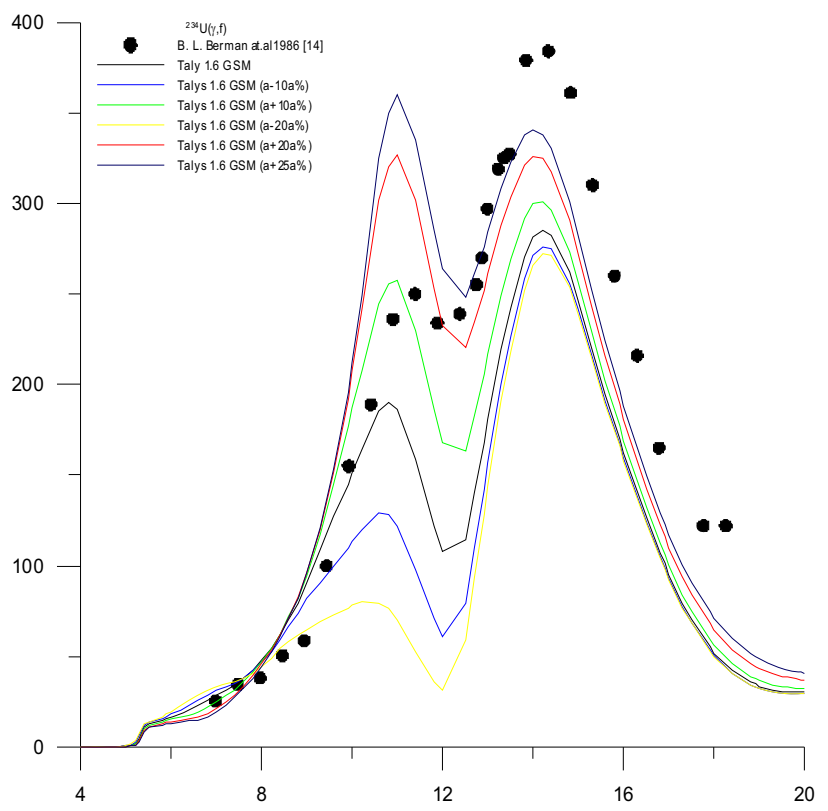

Figure. 4. Theoretical calculations of ${ }^{234} \mathrm{U}(\gamma, \mathrm{f})$ reaction cross section using GSM level density model. The experimental data are taken from EXFOR.

Experimental cross-section values of ${ }^{234} \mathrm{U}(\gamma, \mathrm{f})$ reaction have been compared with theoretically calculated values for three level density models with TALYS 1.6 code in Fig.1.

Experimental cross section values are compared with the theoretically calculated cross section values using CTM, BSFGM and GSM model of TALYS code in Fig. 1. According to Fig. 1, CTM model calculations have similar results with experimental cross section values than other models calculations. Figs. 2-4 have been plotted to show the effects of the level density model parameter on the theoretical calculations for each level density model. The aim of these graphs is to obtain best level density model parameter in each model.

\section{Conclusion}

In this study, it has been showed that how the level density parameter effects theoretical calculations of reaction cross sections depend on the level density models.

CTM model calculations show best compatibility with experimental results and when the similar results obtained by changing a parameter in other models, then level density parameter of that model approaches the value of CTM model parameter.

\section{References}

1. A.J. Koning, S. Hilaire, S. Goriely, TALYS-1.6 (2013) Nuclear Research and Consultancy Group. www.talys.eu

2. Y.S. Perkasa, et al., The 2nd International Conference on Advances in Nuclear Science and Engineering (ICANSE 2009), AIP Conf. Proceedings, Bandung, Indonesia (2010), 288.

3. D. Rochman, M. Herman, P. Oblozinsky, (Brookhaven National Laboratory) Formal Report (BNL-74667) (2005).

4. I. Sirakov, R. Capote, F. Gunsing, P. Schillebeeckx and A. Trkov, Annals of Nuclear Energy 35 (2008), 1223-1231.

5. EXFOR/CSISRS (Experimental Nuclear Reaction Data File) Database, (Brookhaven National Laboratory, National Nuclear Data Center, (2007). http://www.nndc.bnl.gov

6. A. Gilbert, A.G.W. Cameron, Can. J. Phys. 43 (1965) 1446-1496.

7. W. Dilg, et al., Nucl. Phys. A 217(1973)269-298.

8. A.V. Ignatyuk, et al., Phys. Rev. C 47 (4),(1993) 1504-1513.

9. A.V. Ignatyuk, K.K. Istekov, G.N. Smirenkin, Sov. J. Nucl. Phys. 29 (4),(1979)875-883.

10. H. Baba, Nucl. Phys. A 159 (1970) 625.

11. A.V. Ignatyuk, G.N. Smirenkin, A.S. Tishin, Sov. J. Nucl. Phys. 21 (3) (1975) 255.

12. A.J. Koning, S. Hilaire, S. Goriely, Nuclear Physics A 810 (2008) 13-76

13. RIPL, 1998. Reference Input Parameter Library for Theoretical Calculations of Nuclear Reactions http://www-nds.iaea.org/ripl/.

14. B.L. Berman, J.T. Caldwell, E.J. Dowdy, S.S. Dietrich, P. Meyer, and R.A. Alvarez, Phys. Rev. C 34, (1986) 2201. 